\title{
Central Venous Catheter-Related Bloodstream Infection Caused by Staphylococcus aureus: Microbiology and Risk Factors
}

\author{
Geraldo Sadoyma ${ }^{1}$, Augusto Diogo Filho ${ }^{2}$ \\ and Paulo Pinto Gontijo Filho ${ }^{1,2}$
}

\author{
${ }^{1}$ Laboratory of Microbiology; ${ }^{2} \mathrm{CCIH}$ of Clinical Hospital of \\ Federal University of Uberlândia; Uberlância MG, Brazil.
}

\begin{abstract}
Although central vascular catheters (CVC) are indispensable in modern medicine, they are an important risk factor for primary bacteremias. We examined the incidence and risk factors associated with catheter-related bloodstream infection (CR-BSI) caused by Staphylococcus aureus in surgical patients. A prospective study was carried out in the Hospital das Clínicas da Universidade Federal de Uberlândia (HC-UFU) from September 2000 to December 2002. The skin insertion site, catheter tip, and blood were microbiologically analyzed. Demographics and risk factors were recorded for each patient, and cultures were identified phenotypically. Staphylococcus aureus was the most frequent pathogen, with an incidence rate of 4.9 episodes of CR-BSIs per 1,000 catheter/days. Based on logistic regression, the independent risk factors were: colonization on the insertion site $=200$ colony forming units $(\mathrm{CFU}) / 20 \mathrm{~cm}^{2}(\mathrm{p}=0.03$; odds ratio $(\mathrm{OR})=6.89)$ and catheter tip $(\mathrm{p}=0.01 ; \mathrm{OR}=7.95)$. The CR-BSI rate was high; it was mainly associated with $S$. aureus, and skin colonization at the insertion site and on the catheter tip were important risk factors for CR-BSI.
\end{abstract}

Key Words: Staphylococcus aureus, ventral venous catheter, bloodstream infection.

Safe vascular access is one of the key factors for modern medical practice; however, the intravascular devices (IVDs) needed for establishing reliable access are significantly associated with iatrogenic disease, especially bacteremia and candidemia [1,2]. Over 250,000 bloodstream infections (BSI) related to the presence of IVDs occur each year in the U.S., with an attributed death rate of 12-25\%; BSIs also extend hospital internment, with additional costs of US $\$ 33,000-35,000 /$ patient $[3,4]$. Among IVDs, the use of central venous catheters (CVCs) is frequently followed by both local and systemic complications, including septic thrombophlebitis, endocarditis, metastatic infections, and bacteremias [5].

It is estimated that over $80 \%$ of all catheter-related bloodstream infections (CR-BSIs) are associated with CVCs, although they account for only a small percentage of all vascular catheters. CR-BSIs cause substantial morbidity and mortality, and they increase internment time and costs [6].

The pathogenesis of CR-BSI is multifactorial and complex. Although venous and arterial catheters may be colonized via the bloodstream from infections at other sites, through intestinal translocation or through administration of fluids (intrinsic contamination), available data suggest that most infections by staphylococci result from the migration of these microorganisms from the skin insertion site or from the catheter hub [7].

Received on 06 December 2005; revised 22 March 2006.

Address for correspondence: Dr. Geraldo Sadoyama, Área de Imunologia, Microbiologia e Parasitologia, Laboratório de Microbiologia, Bloco 4C, Campus Umuarama, Universidade Federal de Uberlândia, Uberlândia, MG, Brazil. 38400-902. Phone: 55 (34) 3218-2236 Fax number: 55 (34) 3218-2333

E-mail: sadoyama@umuarama.ufu.br gsadoyama@yahoo.com.br

The Brazilian Journal of Infectious Diseases 2006;10(2):100-106. (C) 2006 by The Brazilian Journal of Infectious Diseases and Contexto Publishing. All rights reserved.
According to the "US National Nosocomial Infections Surveillance System Report", the pathogens most frequently associated with CR-BSI etiology from 1991-1999 were: coagulase-negative Staphylococcus (CoNSs) (37\%), S. aureus (13\%), Enterococcus spp. (13\%), and Candida albicans $(8 \%)[8]$.

We evaluate the pathogenesis of primary $S$. aureus bacteremias in surgical patients who had undergone central venous catheterization, and we examined their respective risk factors.

\section{Material and Methods}

Study design. An observational prospective study was carried out through an active search system, based on spontaneous hospital demand, at Clinical Surgical Ward II of the Uberlândia Federal University Hospital (HC-UFU). Demographics, and intrinsic and extrinsic risk factors were recorded for each of the patients.

\section{Microbiological techniques}

CVC insertion site. The skin at the insert site of CVC was swabbed. Two samples were taken: the first, when the catheter was inserted and the second 5-7 days after insertion. Approximately $20 \mathrm{~cm}^{2}$ of skin at the catheter insertion site was cleaned using sterile pre-moistened swabs. The swab was placed into an $1 \mathrm{~mL}$ tube with PBS $+0.1 \%$ of sodium thiosulphate, which agitated with a vortex, and about $0.1 \mathrm{~mL}$ of the fluid was inoculated on blood agar and mannitol salt plates. Skin cultures were considered positive whenever $\geq 200$ UFC were isolated [9].

CVC tip. Catheters were removed under sterile conditions. The tips of the catheters were cut with sterile scissors and 
transported to the laboratory in tubes containing $10 \mathrm{~mL}$ of phosphate-buffered saline (PBS) $+0.1 \%$ Tween 80 . The cultures of the catheter tips were examined quantitatively, using a modified Brun-Buisson [10] technique; a segment of approximately $5 \mathrm{~cm}$ of the catheter tip was placed in a tube containing $10 \mathrm{~mL}$ of PBS $+0.1 \%$ Tween 80 and agitated in a vortex for 1 minute; $0.1 \mathrm{~mL}$ of the liquid was inoculated in agar blood, McConkey agar and mannitol salt agar plates and incubated at $37^{\circ} \mathrm{C}$ for 24 hours to determine the number of colony forming units (CFUs). Cultures were considered positive when $\geq 10^{2} \mathrm{CFU} / \mathrm{mL}$ were detected.

Hemocultures. Blood specimens were obtained through peripheral venous puncture. Hemocultures were performed by inoculating $5-10 \mathrm{~mL}$ of blood into a flask of the commercial automated system Bactec/Alert ${ }^{\circledR}$ (Vitek System, Organon Teknika Corp. ). Positive culture flasks were sub-cultured in MacConkey agar and blood Agar, and plates were incubated at $37^{\circ} \mathrm{C}$ for $24-48$ hours.

Bacterial identification. Clinical specimens obtained from skin at the insertion site of the central vascular catheter tip were identified by classical techniques, being initially separated into Gram-negative and Gram-positive bacilli, Gram-positive cocci and yeast-form fungi and afterwards by their morphological/staining characteristics. Gram-positive cocci were subjected to complementary tests: oxidase, catalase, growth in $\mathrm{NaCl}$, coagulase, mannitol fermentation and DNAse for the identification of $S$. aureus, coagulase-negative Staphylococcus (CoNS) and other Gram-positive cocci. Blood cultures were obtained from the microbiology lab of HC-UFU.

\section{$\underline{\text { Antimicrobial susceptibility tests }}$}

Disk diffusion agar technique. Samples were cultured in TSB medium at $37^{\circ} \mathrm{C}$ for 24 hours and then diluted in saline solution till the suspension opacity corresponded to the 0.5 tube of the MacFarland scale $\left(1-2.10^{8} \mathrm{CFU} / \mathrm{mL}\right)$; they were then seeded with a swab over the medium surface [11]. The following antimicrobial discs were used: amoxacillin-clavulanate, rifampin, clindamycin, cephalothin, tetracycline, sulfametoxazole-trimethoprim, ampicillin, ciprofloxacin, gentamicin, vancomycin, chloramphenicol, erythromycin, quinupristin-dalfopristin, linezolide, and oxacillin. A standard sample of $S$. aureus ATCC 25923 was used as a control for the susceptibility test.

CR-BSI definition. Catheter-related bloodstream infection (CRBSI) were defined as isolation of the same microorganism (i.e. identical species and resistance) from a semiquantitative or quantitative culture of a catheter segment and from the blood (preferably drawn from a peripheral vein) of a patient with accompanying clinical symptoms and no other source of infection.

\section{$\underline{\text { Statistical analysis }}$}

The statistical analysis of risk factors for infection and microbiological results were performed by applyaing the $\div 2$ test for comparing percentage values (qualitative variables) and the Fisher's exact test, when n was equal to or less than five. Risk factors and microbiological results were individually compared against a variable response (univariate analysis) with two by two contingency tables. Multivariate analysis through a logistic regression model were used for variables with high odds ratios. The Student's t-test was used for comparing means (quantitative variables). Statistical significance was defined as a $\mathrm{p}$ value less than 0.05 . The analysis of variables was performed with statistical software SPSS PC version 11.0 (SPSS, Chicago) and Epi Info Software version 2000 (CDC Atlanta).

\section{Results}

Among 198 patients with a central vascular catheter inserted in the jugular vein $(n=84)$ or a subclavia vein $(n=114)$, 19 were withdrawn from the epidemiological analysis due nonrecovery of catheter tips, removal to another unit, or hospital discharge, reducing the study to 179 patients. Four CR-BSIs caused by $S$. aureus were detected, another two were caused by MRSA. The CR-BSI rate caused by $S$. aureus was 4.9 episodes per 1,000 days/catheter and the CVCs colonization was $21.2 \%$.

Risk factors analysis for catheter tip colonization are given Table 1. Evaluation by multivariate logistic regression analysis of risk factors associated with this contamination indicated the following: colonization at the CVC insertion site with $\geq$ $200 \mathrm{CFU} / 20 \mathrm{~cm}^{2}$ skin, internment $\geq 14$ days, catheterization $\geq 7$ days and presence of erythema (Table 2).

Risk factors significantly associated with a CR-BSI: insertion site included: $\geq 200 \mathrm{CFU} / 20 \mathrm{~cm}^{2}, \geq 10^{2} \mathrm{CFU}$ in the CVC tip, and presence of a multilumen catheter (Table 3 ). Based on the multivariate analysis, only bacteria at the insertion site $(\mathrm{p}=0.03 ; \mathrm{OR}=6.89$; confidence interval $(\mathrm{CI})=2.42-21.90)$ and in the tip $(\mathrm{p}=0.01 ; \mathrm{OR}=7.95 ; \mathrm{CI}=1.95-19.60)$ were independent factors for CR-BSI (Table 4).

Microorganisms most frequently seen within SI, PC and blood are listed in Figure 1. The most frequent ones in SI were coagulase-negative Staphylococcus $(49.7 \%)$ and S. aureus (31.2\%), followed by Enterococcus (6.4\%). In the microbiological analysis of the catheter tip, there was predominance of coagulase-negative Staphylococcus $(60.5 \%)$ and $S$. aureus $(28.9 \%)$, followed by Gram-negative bacilli (BGN) (7.9\%). The frequency of isolates of S. aureus in the blood was greater than that of coagulase-negative Staphylococcus (41.4\% versus $37.9 \%$, respectively), followed by BGN (17.2\%) and Enterococcus (3.5\%). One hundred thirtynine samples of $S$. aureus were isolated in these patients, 57 of which $(41.0 \%)$ were MRSA and $82(59.0 \%)$ MSSA. The 
Table 1. Risk factors associated with central venous catheter tip colonization.

\begin{tabular}{|c|c|c|c|}
\hline Risk factors & $\begin{array}{l}\text { Catheter tip positive } \\
\qquad \mathrm{N}=38\end{array}$ & $\begin{array}{l}\text { Catheter tip negative } \\
\qquad N=141\end{array}$ & $\mathbf{P}(\mathbf{R R})$ \\
\hline Dressing & 2 & 14 & $0.52(0.57)$ \\
\hline$\geq 200 \mathrm{CFU} / 20 \mathrm{~cm}^{2}$ at the insertion site & 24 & 53 & $0.008(2.27)$ \\
\hline Age $\geq 60$ years & 23 & 67 & $0.21(1.52)$ \\
\hline \multicolumn{4}{|l|}{ Internment } \\
\hline$\geq 7$ days & 33 & 83 & $0.02(3.58)$ \\
\hline$\geq 14$ days & 31 & 57 & $<0.01(4.58)$ \\
\hline Catheterization $\geq 7$ days & 38 & 65 & $<0.01(\mathrm{NC})$ \\
\hline Antimicrobial use & 30 & 96 & $0.27(1.58)$ \\
\hline$\geq 3$ antimicrobials & 15 & 20 & $0.001(2.68)$ \\
\hline Other invasive devices & 37 & 131 & $0.46(2.42)$ \\
\hline$\geq 3$ devices & 14 & 60 & $0.65(0.83)$ \\
\hline Hypoalbuminamia & 14 & 58 & $0.77(0.87)$ \\
\hline Fever & 24 & 53 & $0.008(2.27)$ \\
\hline Erythema & 16 & 14 & $0.01(3.61)$ \\
\hline Mortality & 05 & 07 & 0.14 (1.98) \\
\hline
\end{tabular}

Table 2. Independent risk factors for catheter tip colonization determined by multivariate logistic regression

\begin{tabular}{lccc}
\hline Risk factors & P Value & Odds Ratio & Confidence interval \\
\hline Insertion site $\geq 200$ CFU/20 $\mathrm{cm}^{2}$ & 0.012 & 2.15 & $1.19-9.98$ \\
Internment $\geq 14$ days & 0.047 & 1.97 & $1.02-8.45$ \\
Catheterization $\geq 7$ days & 0.038 & 1.78 & $1.06-7.98$ \\
Erythema & 0.022 & 2.14 & $2.01-13.61$ \\
\hline
\end{tabular}

Table 3. Risk factors associated with catheter-related bloodstream infection (CR-BSI) in surgical patients of the HC-UFU

\begin{tabular}{|c|c|c|c|}
\hline \multirow[t]{2}{*}{ Risk factors } & \multicolumn{2}{|c|}{ CR-BSI } & \multirow[t]{2}{*}{ Pvalue (Relative Risk) } \\
\hline & Presence $n=04$ & Absence $\mathrm{n}=175$ & \\
\hline Dressing & 0 & 16 & $1.0\left(\mathrm{NC}^{*}\right)$ \\
\hline Insertion site $\geq 200 \mathrm{CFU} / 20 \mathrm{~cm}^{2}$ & 4 & 65 & $0.02(\mathrm{NC})$ \\
\hline Catheter tip $\geq 10^{2} \mathrm{CFU}$ & 4 & 34 & $0.001(\mathrm{NC})$ \\
\hline Multilumen catheter & 3 & 28 & $0.016(14.32)$ \\
\hline Age $\geq 60$ years & 1 & 89 & $0.36(0.33)$ \\
\hline \multicolumn{4}{|l|}{ Internment } \\
\hline$\geq 7$ days & 4 & 109 & $0.29(\mathrm{NC})$ \\
\hline$\geq 14$ days & 3 & 85 & $0.36(3.1)$ \\
\hline Catheterization $\geq 7$ days & 4 & 99 & $0.13(\mathrm{NC})$ \\
\hline Antimicrobial use & 4 & 122 & $0.32(N C)$ \\
\hline$\geq 3$ antimicrobials & 2 & 33 & $0.17(4.11)$ \\
\hline Others invasive devices & 4 & 164 & $1.0(\mathrm{NC})$ \\
\hline$\geq 3$ devices & 3 & 71 & $0.30(4.26)$ \\
\hline Hypoalbuminamia & 4 & 68 & $0.02(\mathrm{NC})$ \\
\hline Fever & 4 & 73 & $0.03(\mathrm{NC})$ \\
\hline Erythema & 2 & 28 & $0.13(4.97)$ \\
\hline Mortality & 0 & 12 & $1.0(\mathrm{NC})$ \\
\hline
\end{tabular}

*Not calculated. 
Table 4. Independent risk factors associated with CR-BSI by multivariate logistic regression

\begin{tabular}{lccc}
\hline Risk Factors & P & Odds Ratio & C \\
\hline$\geq 200 \mathrm{CFU} / 20 \mathrm{~cm}^{2}$ at the insertion site & 0.03 & 6.89 & $2.42-21.90$ \\
Catheter tip $\geq 10^{2} \mathrm{CFU}$ & 0.01 & 7.95 & $1.95-19.60$ \\
\hline
\end{tabular}

$\mathrm{CI}=$ confidence interval.

Table 5. Antibiotic resistance patterns of MRSA and MSSA bacterial isolates from patients of the HC- UFU

\begin{tabular}{lccc}
\hline Antimicrobials & MRSA n=57(\%) & MSSA n=82(\%) & Pvalue \\
\hline Ampicillin & $54(94.7)$ & $57(69.5)$ & $<0.001$ \\
Amoxacillin-clavulanate & $30(52.6)$ & $21(25.9)$ & 0.002 \\
Cephalothin & $44(77.2)$ & $21(25.6)$ & $<0.001$ \\
Ciprofloxacin & $57(100)$ & $40(48.7)$ & $<0.001$ \\
Clindamycin & $57(100)$ & $2.4(2)$ & $<0.001$ \\
Chloramphenicol & $57(100)$ & $12.2(10)$ & $<0.001$ \\
Quinupristin-dalfopristin & $0(0)$ & $0(0)$ & 1.0 \\
Erythromycin & $57(100)$ & $41(50.0)$ & $<0.001$ \\
Gentamicin & $57(100)$ & $3.6(3)$ & $<0.001$ \\
Linezolide & $0(0)$ & $0(0)$ & 1.0 \\
Rifampin & $57(100)$ & $7(8.5)$ & $<0.001$ \\
Sulfametoxazole-trimethoprim & $57(100)$ & $7(8.5)$ & $<0.001$ \\
Tetracycline & $48(84.2)$ & $10(12.2)$ & $<0.001$ \\
Vancomycin & $0(0)$ & $0(0)$ & 1.0 \\
\hline
\end{tabular}

Figure 1.

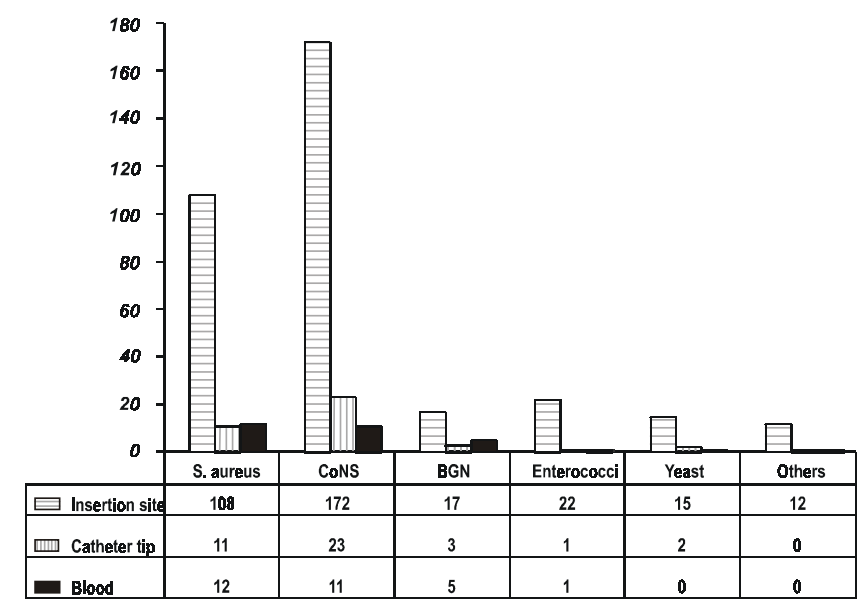

phenotype MRSA was responsible for $37.1 \%$ and $36.4 \%$ of colonies at the insertion site and in the catheter tip, respectively, and they accounted for $50 \%$ of the BSIs caused by S. aureus.

Among MRSA isolates, multiresistance was the most commonly observed pattern, with simultaneous resistance to clindamycin, chloramphenicol, erythromycin, gentamycin, rifampicin, sulfametoxazol-trimetoprim, and tetracycline. Among MSSA samples, resistance to ampicillin, erythromycin and ciprofloxacin were more frequent. All the samples of $S$. aureus (MRSA/MSSA) were susceptible to dalfopristin/quinupristin and linezolide, as well as to vancomycin (Table 5).

\section{Discussion}

The incidence of CR-BSI varies considerably with the type of catheter, but most $(90.0 \%)$ are associated with the use of short-term, non-tunnel CVCs, inserted into the internal jugular vein and the subclavia vein [12], which are the veins that we analyzed. The proportion of CB-BSIs varies from $2.5 \%$ to $6.4 \%$ [13-16], with rates corresponding to 2.4-12.0 episodes per 1,000 days/CVC [17-22]. We found a rate of $2.2 \%$, or 4.9 episodes per 1,000 days/catheter, among patients that were not in the intensive-care unit (ICU).

A series of studies published in the 90's investigating CVCs that were not soaked with antimicrobial antiseptic gave a variation of the colonization rate for these catheters of from $23.6 \%$ to $52.2 \%$ in patients in ICUs [14-16,23-25]. In our study the colonization rate of CVCs was lower (21.2\%).

Risk Factors associated with CR-BSI are numerous; they can be divided into intrinsic and extrinsic factors, with the following highlights: surgical services, prolonged hospital internment, ICU care, active infection at another site, underweight premature born infant, difficulty in inserting the $\mathrm{CVC}$, high APACHE score, site of CVC insertion (internal jugular/femoral veins), type of bandage, skin colonization at the pericatheter site, duration of catheterization (>7/10 days), colonization of the catheter cannon, parenteral feeding, among 
others $[22,26]$. We found the following factors of risk to be significantly associated with CR-BSI: SI colonization $\geq 200$ $\mathrm{CFU} / 20 \mathrm{~cm}^{2}$ and $\geq 10^{3} \mathrm{CFU}$ on the catheter tip.

Studies have shown that an important factor of risk associated with PC and cannon colonization, is colonization of the pericatheter cutaneous site [27,28]. This colonization proved to be an independent risk for factor PC colonization in our study, as were internment time greater than or equal to 14 days and catheterization over seven days.

In CVCs lasting for less than eight days, catheter colonization is more commonly ( $75 \%$ to $90 \%$ ) a result of extralumen migration of skin microorganisms to the catheter tip inside the blood vessel [22]. Colonization at the insertion site of 6.5-56.5 results in a relative risk (RR) for CR-BSI [26]. This observation suggests that bandages applied at this site can have considerable influence on the incidence of these infections [2931]. In most infirmaries and units of the HC-UFU non-use of frequent occlusive bandaging prevails in this invasive procedure. Only 16 patients $(8.9 \%)$ had occlusive bandages and $87.5 \%$ of them had $<200 \mathrm{CFU} / 20 \mathrm{~cm}^{2}$ on the pericatheter skin.

Most of the microorganisms implied in CR-BSI are part of the normal skin microbiota. Gram-positive cocci are responsible for, at least two-thirds of these infections [32]. Coagulasenegative Staphylococcus (Staphylococcus epidermidis) is the most common agent, followed by $S$. aureus, Enterococcus spp., Gram-negative bacilli and yeast-form fungi [33]. However, in our study $S$. aureus was the most frequent pathogen (38\%), surpassing CoNS (34.0\% and BGN (24.0\%).

In an overview published in 2002 [34] about a series of prospective studies performed in the $90 \mathrm{~s}$, CoNS were the most frequently isolated microorganisms on the catheter tip, different from the blood, where the $S$. aureus was the most frequent. More recent studies confirm these findings [28,35]. In our investigation, CoNs frequently colonized the tip was (60\%), while $S$. aureus was found in $30 \%$; in the blood we saw a higher percentage of $S$. aureus (38\%). The prevalence of $S$. aureus over CoNS in the blood was also reported by other investigators [36-38].

The microbiological diagnosis of a CR-BSI is very important, because its therapy will vary according to the isolated agent and according to its resistance spectrum. CoNS result in lower mortality rates than $S$. aureus, BGN, and Candida spp.[39,40].

Another important aspect about these infections is resistance to antimicrobial drugs, since many CR-BSI agents are resistant to routinely-used antimicrobials. Oxacillinresistant CoNS and $S$. aureus infections are growing in frequency, especially within tertiary and/or school hospitals; they account for over $30 \%$ of isolates in some Brazilian hospitals, 34\% in North-American hospitals and about 1.8$54.0 \%$ in European countries [41,42]. Among CoNS samples, isolates resistant to penicillin reach rates up to $85.5 \%$ [43]. In a previous study carried out at the HC-UFU, we found a frequency of $44 \%$ samples resistant to oxacillin amongst $S$. aureus isolates [44], similar to the $41.0 \%$ that we now have found.

MSRA isolates are usually resistant to several antibiotics, including betalactamics, aminoglucosides, macrolides, fluoroquinolones, chloramphenicol, mupirocin, and others; vancomycin is the drug of choice for treating severe infections by these microorganisms [45]. Recently, two new drugs, dalfopristine/quinopristine and linezolide, appeared as options for treating infections caused by MRSA [46]. In our investigation, we found an association of resistance to oxacillin in MRSA isolates showing susceptibility to vancomycin, dalfopristine/quinopristine and linezolide.

The routes of contamination by staphylococci of the CVC tip, with subsequent CR-BSI, are extraluminal in those of short lasting ( $<8$ days) and intraluminal in those of long lasting $(>8$ days) infections; the blood stream is the most probable route in critically ill patients, as previously described [47]. In our study, the presence of microorganisms at the CVC insertion site was an independent risk factor associated with CVC colonization, showing the importance of the skin as a reservoir of this microorganism for CVC-tip colonization. In CR-BSI pathogenesis, while $S$. epidermidis and other CoNS usually adhere to the surface of the CVC polymer to form slime/ glycocalyx after catheter insertion into the vascular system, in $S$. aureus the main mechanism of invasion is mediated by protein adhesins on the bacterial cell wall, involving fibrinogenic receptors and fibronectine in the biofilm of the CVC tip [48].

In the pathogenesis of CR-BSIs caused by S. aureus, the importance of nasal contamination by this microorganism is well established [49,50]. Sesso et al. [51] showed that nasal decolonization reduces the risk of pericatheter colonization eight-fold and CR-BSI four-fold. Nasal colonization by $S$. aureus in patients enrolled in our study was not assessed as a predisposing factor for this infection, but the presence of $\geq 200 \mathrm{CFU} / 20 \mathrm{~cm}^{2}$ at the $\mathrm{CVC}$ insertion site was an independent risk factor for $\mathrm{CR}-\mathrm{BSI}(\mathrm{OR}=6.89)$. The bacterial load on the CVC tip is related to CVC culture positivity, and to finding of bacteria in blood culture and therefore to CR-BSI [52]. In our study, considering only cases of sepsis by $S$. aureus, $\geq 10^{2}$ CFU catheter tip colonization was a significant factor $(\mathrm{OR}=7.85)$, based on multiple logistic regression analysis.

The potential risk of not using occlusive bandages in the routine care of $\mathrm{CVCs}$, which we found frequently in our investigation, also influences colonization levels at the CVC insertion site; it is associated with colonization on the catheter tip by $S$. aureus, an important factor in the pathogenesis of primary bacteremias.

\section{References}

1. Raad I. Intravascular catheter-related infections. The Lancet 1998;351:893-8.

2. Mermel L.A. Prevention of intravascular catheter-related infections, Annals Internal Medicine 2000;132:391-402. 
3. Pitet D., Tarara D., Wenzel, R. Nosocomial bloodstream infection in critically ill patients: excess length of saty, extra costs, and atributable mortality. Journal American Medical Association 1993;271:1598-1601.

4. Digiovine B., Chenoweth C., Watts C., Higgins M. The atributable mortality and costs of primary nosocomial bloodstream infections in intensive care unit. American Journal of Respiratory Critical Care Medicine 1999;160:976-81.

5. Fratino G., Mazzola C., Buffa P., et al. Mechanical complications related to indwelling central venous catheter in pediatric hematology/oncology patients. Pediatric Hematology and Oncology 2001;18:317-24.

6. Maki D.G. Infections due to infusion therapy. In: Bennett J.V., Brachman P.S.[ eds.] Hospital Infections. Boston, MA: Little Brown and Co. 1998;689-724.

7. Crump J.A., Collignom P.J. Intravascular Catheter-associated infections. European Journal Clinical of Infectious Diseases 2000; $19: 1-8$.

8. National Nosocomial Infections Surveillance (Nnis) System Report, Data Summary from January 1992-June 2001, issued August 2001. American Journal Infection Control 2000;29:404-21.

9. Maki D.G., Ringer M.,Alvarado C.J. Prospective randomised trial of povidine-iodine, alcohol, and chlorexidine for prevention of infection associated with central venous and arterial catheters. Lancet 1991;338:339-43.

10. Brun-Buisson C., Abrouk F., Legrand P., et al. Diagnosis of central venous catheter-related sepsis: Critical level of quantitative tip cultures. Archives Internal Medicine 1987; $147: 873-7$.

11. National Committee For Clinical Laboratory Standards. Performance Standards for Antimicrobial Disk Susceptibility Tests, v. 17, 1997.

12. Kluger D., Maki D. The relative risk of intravascular devicerelated bloodstream infections with different types of intravascular devices in adults: a meta-analysis of 206 published studies. Infection Control Hospital Epidemiology 2000;21:95-6.

13. Bach A., Schmidt H., Bottiger B., Vogel B. Retention of antibacterial activity and bacterial colonization of antisepticbonded central venous catheters. Journal Antimicrobial Chemotherapy 1996;37:315-22.

14. Tennenberg S., Lieser M., Mccurdy B., et al. A prospective randomized trial of an antibiotic- and antiseptic-coated central venous catheter in the prevention of catheter-related infections. Archives Surgery 1997;132:1348-51.

15. Heard S.O., Wagle M., Vijayakumar E. Influence of triple-lumen central venous catheters coated with chlorhexidine and silver sulfadiazine on the incidence of catheter-related bacteremia. Archives Internal Medicine 1998;158:81-8.

16. Hannan M., Juste R.N., Umasanker S. Antiseptic-bonded central venous catheters and bacterial colonisation. Anaesthesia 1999;54:868-72.

17. Barsic B., Beus I., Marton E., et al. Nosocomial infections in critically ill infectious disease patients: results of a 7-year focal surveillance. Infection 1999;27:16-22.

18. Wallace W.C., Cinat M., Gornick W.B., et al. Nosocomial infections in the surgical intensive care unit: a difference between trauma and surgical patients. American Surgeon 1999;.65:987-90.
19. Eggimann P., Harbarth S., Constantin M.N., et al. Impact of a prevention strategy targeted at vascular-access care on incidence of infections acquired in intensive care. Lancet 2000;355:1864-8.

20. Finkelstein R., Rabino G., Kassis I., Mahamid I. Deviceassociated, device-day infection rates in an Israeli adult general intensive care unit. Journal Hospital Infection 2000; $44: 200-5$.

21. Richards M.J., Edwards J.R., Culver D.H., Gaynes R.P. Nosocomial infections in combined medical-surgical intensive care units in the United States. Infection Control Hospital Epidemiology 2000;21:510-5.

22. Sheretz J.R. Pathogenesis of vascular catheter infection, p. 111125. In F.A. Waldvogel, A.L. Bisno (eds). Infections Associated with Indewelling Medical Devices. 3rd ed. ASM Press, Washington, D.C. 2000.

23. Loo S., Van Heerden P.V., Gollege C.L., et al. Infection in central lines: antisepticimpregnated versus standard non-impregnated catheters. Anaesthesia Intensive Care 1997;25:637-9.

24. Maki D.G., Stolz S.M., Wheeler S., Mermel L.A. Prevention of central venous catheter-related bloodstream infection by use of an antiseptic-impregnated catheter. A randomized, controlled trial. Annals Internal Medicine 1997;127:257-66.

25. Marik P.E., Abraham G., Careau P., et al. The ex vivo antimicrobial activity and colonization rate of two antimicrobial-bonded central venous catheters. Critical Care Medicine 1999;27:1128-31.

26. Safdar N., Kluger D.M., Maki D.G. A review of risk actors for CR-BSI caused by percytaveaously inserted, noncuffed central venous catheter. Medicine 2002;81:466-79.

27. Egebo K., Toft P., Jakobsen C.J. Contamination of central venous catheters. The skin insertion site wound is a major source of contamination. Journal Hospital Infection 1996;32:99-104.

28. Mahieu L.M., Dedooy J.J., Muynck A.O., et al. Microbiology na drisk factors for catheter exit-ste and -hub colonization in neonatal intensive care unit patients. Infection control Hospital Epidemiology 2001;22:357-62.

29. Engervall P., Ringertz S., Hagman E., et al. Change of central venous catheter dressings twice a week is superior to once a week in patients with haematological malignancies. Journal Hospital Infection 1995;29:275-86.

30. Nikoletti S., Leslie G., Gandossi S., et al. A prospective, randomized, controlled trial comparing transparent polyurethane and hydrocolloid dressings for central venous catheters. American Journal Infection Control 1999;27:488-96.

31. Curchoe R.M., Powers J., El-Daher N. Weekly transparent dressing changes linked to increased bacteremia rates. Infection Control Hospital Epidemiology 2002;23:730-2.

32. Darouiche R.O., Raad I.I., Heard S.O., Mayhall G. A comparison of two antimicrobial-impregnated central venous catheters. New England Journal Medicine 1999;340:1-8.

33. Centers For Disease Control And Prevention. Staphylococcus aureus resistant to Vancomycin - US. MMWR 2000;48:1165-7.

34. Raad I.I., Hanna H.A. Intravascular catheter-related infections. Archives Internal Medicine 2002;162:871-8.

35. Dobbins B.M., Kite P., Kindon A., et al. DNA fingerprinting analysis of coagulase negative staphylococci implicated in catheter related bloodstream infections. Journal Clinical Pathology 2000;55:824-8. 
36. Bantar C., Bustos J.L., Vesco E., Morera G. Central venous catheter-related infection: A prospective, observational study to assess the incidence rate at a teaching hospital in Argentina. Infection Control Hospital Epidemiology 2002;23:757-8.

37. Richards B., Chaboyer W., Bladen T., Schluter P.J. Effect of central venous catheter type on infections: a prospective clinical trial. Journal Hospital Infection 2003;54:10-7.

38. Öncü S., Özsüt H.,Yldirim Y., et al. Central venous catheter related infections: Risk factors and the effect of glycopeptide antibiotics. Annals of Clinical Microbiology and Antimicrobials 2003;2:1-6.

39. Valles J., Leon C., Alvarez-Lerma F. Nosocomial bacteremia in critically ill patients: a multicenter study evaluating epidemiology and prognosis. Spanish Collaborative Group for Infections in Intensive Care Units of Sociedad Espanola de Medicina Intensiva y Unidades Coronarias (SEMIUC). Clinical Infectiuos Diseases 1997;24:387-95.

40. Rello J. Evaluation of outcome of intravenous catheter-related infections in critically ill patients. Am J Respir Crit Care Méd 2000;162:1027-30.

41. Moreira M., Medeiros E.A.S., Pignatari A.C.C., et al. Efeito da infecção hospitalar da corrente sanguínea por Staphylococcus aureus resistente à oxacilina sobre a letalidade e o tempo de hospitalização. Revista Associação Médica Brasileira 1998; $44: 263-8$.

42. Diekema D.J., Pfaller M.A., Jones R.N., et al. Survey of infections due to Staphylococcus species: frequency of occurrence and antimicrobial susceptibility of isolates collected in the United States, Canada, Latin America, Europe, and the Western Pacific Region for the SENTRY antimicrobial Surveillance Program, 1997-1999. Clinical Infectious Diseases 2001;02:S114-32.
43. Frebourg N.B., Cauliez B., Lemeland J.F. Evidence for nasal carriage of methicillin-resistant staphylococci colonizing intravascular devices. Journal of Clinical Microbiology 1999;37:1182-5.

44. Sadoyama G., Gontijo Filho P.P. Risk factors for methicillinresistant and sensitive Staphylococcus aureus in a Brazilian university hospital. Braz J Infect Dis 2000;4:135-43.

45. Chambers H.F. Methicillin Resistance in Staphylococci: Molecular and Biochemical Basis and Clinical Implications. Clinical Microbiology Reviews 1997;10:781-91.

46. Bhavnani S.M., Ballow C.H. New agents for Gram-positive bacteria. Current Opinion in Microbiology 2000;03:528-34.

47. Crnich C.J., Maki D.G. The promise of novel technology for the prevention of intravascular device-related bloodstream infection. Pathogenesis and short-term devices. Clinical Infectious Diseases 2002;34:1232-42.

48. Eiff C.V., Peters G., Heilmann C. Pathogenesis of infections due to coagulase-negative staphylococci. Lancet Infectious Diseases 2002;02:677-85.

49. Kluytmans J., Belkum A.V., Verbrugh H. Nasal Carriage of Staphylococcus aureus: Epidemiology, Underlying Mechanisms, and Associated Risks. Clinical microbiology reviews 1997;10:505-20.

50. Livesly M.A., Tebbs S.E., Moss M.A., et al. Use pulsed field gel eletrophoresis to determine the source of microbial contamination of central venous catheters. European Journal Clinical Infectious Diseases 1998; $17: 108-12$.

51. Sesso R., Barbosa D., Leme I.L., et al. Staphylococcus aureus prophylaxis in hemodialysis patients during central venous catheters: effect of mupirocin ointment. Journal Medical Society Neprhology 1998;09:1085-92.

52. Rijnders B.J.A., Wijngaerden E.V., Peetermans W.E. Cathetertip colonization as a surrogate end point clinical studies on catheter-related bloodstream infection: How strong is the evidence? Clinical Infectious Diseases 2002;35:1053-8. 